\title{
NECESIDADES DE CAPACITACIÓN DE LAS PYMES DEL CANTÓN DE BAGACES
}

\section{TRAINING NEEDS OF SMES BAGACES CITY}

\author{
Luis Alberto Bermúdez Carrillo ${ }^{l}$ \\ luis.bermudez@ucr.ac.cr
}

Fecha de recepción: 5 agosto 2013 - Fecha de aceptación: 29 abril 2014

\begin{abstract}
Resumen
Cuando se mencionan las necesidades que tienen las pequeñas y medianas empresas (pymes) muchas veces se piensa en las que tienen que ver con financiamiento como la máxima; sin embargo, otras limitaciones o carencias pueden constituir factores fundamentales que les impidan poder desarrollar su potencial de una manera estratégica y precisa. Bajo este concepto, el presente estudio tiene como objetivo detectar las necesidades de capacitación que tengan las pymes del cantón de Bagaces, como prioritarias. Por sus características, esta investigación es de tipo descriptivo y está basada en la opinión de una muestra de 98 microempresarios a los que se les aplicó una encuesta para conocer sus opiniones. En general, según los resultados de esta consulta, la mayor cantidad de necesidades de capacitación detectadas tienen que ver con soporte legal y tributario, modelos de gestión y desarrollo organizacional, aspectos tecnológicos y aspectos de índole estructural.

Palabras claves: Pymes, necesidades de capacitación, productos, servicios.
\end{abstract}

\begin{abstract}
When the needs of Small and Medium enterprises ( SMEs ) are mentioned often think of that have to do with financing as low, however, deficiencies or other limitations may constitute key factors that prevent them to develop their potential a convenient and accurate way. Under this concept, the present study aims to identify the training needs with SMEs Bagaces city as priority. Due to its characteristics, this research is descriptive and is based on the opinions of a sample of 98 entrepreneurs to whom they were given a survey to find out their views. In general, according to the results of this survey, the largest number of identified training needs have to do with legal and tax support, management models and organizational development, technological and structural aspects of nature.
\end{abstract}

Key Words: SMEs, training needs, products, services.

1 Docente y Coordinador Carrera Dirección de Empresas, Sede Guanacaste, Universidad de Costa Rica. 


\section{INTRODUCCIÓN}

A pesar de que en Costa Rica se clasifica de distintas maneras a las pequeñas y medianas empresas (microempresas), la clasificación oficial es la del Ministerio de Economía, Industria y Comercio (MEIC), (1997), tal como se publica en el diario oficial La Gaceta N. ${ }^{\circ} 213$ del 5 de noviembre de 1997:

Cuadro 1

Clasificación de las pymes

\begin{tabular}{lccc}
\hline & Número de empleados & $\begin{array}{c}\text { Inversión en maquinaria } \\
\text { y E(e)quipo }\end{array}$ & Ventas anuales \\
\hline Pequeña empresa & $6-30$ & Hasta $\$ 250.000$ & Hasta $\$ 500.000$ \\
Mediana empresa & $31-100$ & Hasta $\$ 500.000$ & Hasta $\$ 1.000 .000$ \\
Pymes & $6-100$ & Hasta $\$ 500.000$ & Hasta $\$ 1.000 .000$ \\
\hline
\end{tabular}

Fuente: Elaboración propia con datos del MEIC.

Como se observa en el cuadro anterior, son pequeñas y medianas empresas las que cuentan con menos de 100 empleados y tienen niveles de facturación en ventas anuales de hasta $\$ 1.000 .000$.

Según datos del Ministerio de Economía Industria y Comercio (MEIC) (2013), en el 2013 las pymes aportaron 46\% de los empleos que había en Costa Rica. Por su parte, el sector privado está integrado por $96 \%$ de esta figura económica.

Asimismo, en dicho reporte se reconoce que las pymes son importantes para la economía y para el desarrollo del país por el número de empleos que aportan y porque con eso contribuye al desarrollo de su economía.

Además, ahí se destaca la importancia de apoyar y fortalecer a las pymes para que su impacto sea mayor y más positivo en la economía nacional. No obstante, en otros estudios consultados no se analizan de manera formal sus necesidades de capacitación. Se revisaron muchos comentarios y argumentaciones de profesionales basadas en estadísticas en los que se habla de que sus efectos son positivos; pero en casos de análisis como este, se siente que requieren capacitación. Si bien es cierto, las pymes son necesarias para el desarrollo de la economía del país, (pero) aún no están totalmente cubiertas ni respaldadas por programas, legislación y decretos que incentiven su creación para el cumplimiento de sus funciones.

Así pues, el cantón de Bagaces fue el elegido para el estudio de sus pymes por dos razones. La primera es que de ellas se contaba con información de primera mano acerca de sus necesidades de capacitación, la cual fue obtenida por medio de la encuesta aplicada a los correspondientes microempresarios. La segunda razón es que también se disponía de datos de indicadores de Bagaces extraídos de la página (http:// www.tse.go.cr/pdf/ficheros/bagaces.pdf, 2013), en la que se indica:

Bagaces es uno de los cantones con peor ubicación en todos sus indicadores: un elevado nivel de pobreza, una alta desigualdad entre hombres y mujeres, un bajo desarrollo humano, una alta vulnerabilidad infantil, un deficiente nivel de competitividad y una deficiente gestión municipal en la zona.

Tales datos permitieron establecer un punto de partida importante para determinar los motivos de esos indicadores, y para dejar constancia de que es necesario hacer algo para lograr que esos índices mejoren y permitan el desarrollo económico del cantón. 
De acuerdo con el anterior planteamiento, la pregunta de investigación que cabe es: ¿Qué tipos de capacitación requieren las pymes del cantón de Bagaces para lograr un mayor nivel de desarrollo y de aporte a la economía local?

En procura de una respuesta a esa interrogante se plantearon los siguientes objetivos de investigación:

- Identificar las principales características de las pymes del cantón de Bagaces.

- Analizar las características de los propietarios de pymes del cantón de Bagaces.

- Describir las actividades económicas desarrolladas por las pymes del cantón de Bagaces y su forma de comercialización.

- Identificar las principales necesidades de capacitación de las pymes del cantón de Bagaces.

Como se mencionó anteriormente, el ámbito de la investigación fue el cantón de Bagaces, específicamente del distrito de Bagaces, a partir de información de la página www.skyscrapercity. com (2013), en la que como primera referencia se comenta:

En la época precolombina el territorio que actualmente corresponde al cantón Bagaces, un grupo de indígenas denominados nahua o azteca, tuvieron un enclave o colonia en la región.

Bagaces en el transcurso de su existencia como asentamiento humano, ha tenido tres ubicaciones. La primera en el área próxima a la confluencia de los ríos Tenorio y Curubicí (hoy Corobicí), en jurisdicción del actual cantón Cañas, en donde se erigió una ermita en 1687, con el propósito de formar una población con los habitantes del valle de Bagaces, sin lograr resultados positivos. La segunda se ubicó hacia el noroeste de la primera, en el sector aledaño a la margen oeste del río conocido hoy como Villa Vieja por el año de 1739. La última se efectuó en 1790 , llevado a cabo por el padre Nicolás Carrillo a su asiento definitivo, en la actual ciudad Bagaces.

Por otro lado, en la página de la Municipalidad de Bagaces (2013), se indica sobre el cantón de Bagaces:

En la época precolombina el territorio que actualmente corresponde al cantón Bagaces, un grupo de indígenas denominados nahua o azteca, tuvieron un enclave o colonia en la región.
Bagaces en el transcurso de su existencia como asentamiento humano, ha tenido tres ubicaciones. La primera en el área próxima a la confluencia de los ríos Tenorio y Curubicí (hoy Corobicí), en jurisdicción del actual cantón Cañas, en donde se erigió una ermita en 1687, con el propósito de formar una población con los habitantes del valle de Bagaces, sin lograr resultados positivos. La segunda se ubicó hacia el noroeste de la primera, en el sector aledaño a la margen oeste del río conocido hoy como Villa Vieja por el año de 1739. La última se efectuó en 1790, llevado a cabo por el padre Nicolás Carrillo a su asiento definitivo, en la actual ciudad Bagaces.

La primera ermita se construyó en 1687. La parroquia se erigió en 1790, dedicada a la virgen de la Inmaculada Concepción. La iglesia actual se construyó en 1940, durante el arzobispado de Monseñor don Víctor Manuel Sanabria Martínez, segundo Arzobispo de Costa Rica; la cual en este momento es sufragada por la Diócesis de Tilarán de la Provincia Eclesiástica de Costa Rica.

\section{METODOLOGÍA}

Para la realización del presente estudio se utilizó como metodología de investigación la descriptiva, basada en los datos de la encuesta aplicada a una muestra de 98 unidades estratégicas pertenecientes al cantón de Bagaces. La finalidad era obtener datos sobre las características de las pymes del cantón y sobre sus necesidades de capacitación. La información recabada se resumió minuciosamente para posteriormente analizarla, a fin de extraer de ella generalizaciones significativas que contribuyeran al conocimiento de cuáles son esas necesidades de capacitación.

Según Best (1982: 91), la investigación descriptiva (se) refiere minuciosamente a lo que es. Está relacionada a condiciones o conexiones existentes; prácticas que prevalecen, opiniones, puntos de vista o actitudes que se mantienen; procesos en marcha; efectos que se sienten o tendencias que se desarrollan. A veces la investigación descriptiva concierne a cómo lo que es o lo que existe se relaciona con algún hecho precedente, que haya influido o afectado una condición o hechos presentes.

Por eso, el muestreo, por ser intencional y por la conveniencia de estudiar únicamente a las microempresas del cantón de Bagaces, específicamente las del distrito de Bagaces, se constituye en un muestreo no probabilístico. 
Al respecto, Grande Esteban y Abascal Fernández (2011) comentan:

En él las unidades muestrales no se seleccionan al azar, sino que son elegidas por las personas. Los diversos tipos de muestreo no probabilístico tienen las siguientes características comunes:

- La selección de la muestra no es aleatoria, sino que se basa, en parte, en el juicio del entrevistador o del responsable de la investigación.

- No se basa en ninguna teoría de la probabilidad y, por lo tanto, no es posible calcular la precisión o acotar el error cometido.

- No es posible calcular estos errores ni la confianza de las estimaciones que, además, no siempre se reducen aumentando el tamaño de la muestra.

- En el muestreo no probabilístico los costes y la dificultad del diseño son más reducidos (al no ser necesario disponer de un marco). Este muestreo puede dar buenos resultados, pero también apareja el riesgo de proporcionar una información errónea. (p. 258)

\section{RESULTADOS}

El resultado de la encuesta aplicada a los 98 microempresarios estudiados del cantón de Bagaces, mostró que todos reunían los requisitos indispensables para ser microempresarios, o al menos mostraron tener una idea productiva.

El análisis se hizo con base en los cuatro objetivos propuestos, organizados en la encuesta para seguir el siguiente orden: identificación de la empresa en lo que compete a la organización legal, conocimiento de las exigencias legales y administrativas de funcionamiento, condiciones legales de sus establecimientos, obtención de recursos y acuerdos de cooperación.

El aspecto de organización legal se refiere si la empresa fue constituida como persona física o como persona jurídica. Física es cuando la empresa está a cargo y bajo la responsabilidad de una única persona, y jurídica cuando la empresa la constituye o representa varias personas.
Además, como segundo punto se analizaron las características del propietario, como la edad, el sexo, el estado civil y el grado de escolaridad.

Venta de bienes o servicios, o ambos, o existencias. Se enfatiza el tipo de servicio o producto que comercializan los microempresarios, y la forma en que lo hacen.

Como cuarto punto, relacionado con el objetivo principal de la investigación, se detallaron las necesidades de capacitación exploradas.

\section{Identificación de la empresa}

Según los resultados, 94 de los microempresarios y empresarias están organizados bajo la figura legal de persona física y cuatro como persona jurídica, específicamente como sociedades anónimas. A pesar de tener actividad económica, las empresas -especialmente las de representación física- no tienen idea de las exigencias legales y administrativas aplicables a sus negocios. Cabe resaltar que de los 98 microempresarios, únicamente 45 tienen conciencia de la obligación que tienen ante instituciones como la CCSS, el INS y el Ministerio de Hacienda, ni de las responsabilidades que como empresarios tienen.

Por un lado, sobre la propiedad o el espacio en el que realizan las actividades, se les consultó si el local es alquilado, propio o si realizan su actividad en forma ambulante, o de qué otra forma.

De los consultados 5\% no respondieron, $36 \%$ manifestaron que es alquilada, $8 \%$ dijeron realizar la actividad en forma ambulante (ni alquilada ni propia) y $51 \%$ trabajan en sus propios locales.

Por otro lado, en lo relativo a las fuentes de financiamiento a las que recurren cuando lo necesitan, $96 \%$ de los entrevistados indicaron que trabajan con recursos propios, y $4 \%$ dijeron obtener los recursos de instituciones gubernamentales, especialmente de bancos estatales. Sin embargo, no se encontró información sobre la utilización de financiamiento proveniente de organismos privados.

Para concluir con lo correspondiente a este apartado, se les preguntó a los entrevistados 
si han suscrito acuerdos de cooperación con otras empresas o instituciones para realizar actividades de innovación y desarrollo de la empresa. Del total de los encuestados $94 \%$ contestaron de manera negativa y $6 \%$ afirmaron haber sido beneficiados con algún tipo de incentivo.

\section{Características del microempresario}

Acerca de la edad de los propietarios o propietarias de pymes, según el cuadro, $70 \%$ tienen entre 31 y 50 años, y se destaca que el grupo de edad menor de 20 años no tiene representación como propietario de pymes.

Cuadro 2

Edad promedio de los microempresarios

\begin{tabular}{ccc}
\hline Años & Cantidad de Microempresarios & Composición porcentual \\
\hline menos de 20 & 0 & $0 \%$ \\
20 a 30 & 16 & $16 \%$ \\
31 a 40 & 39 & $40 \%$ \\
41 a 50 & 29 & $30 \%$ \\
Mayor de 50 & 14 & $14 \%$ \\
Totales & 98 & $100 \%$ \\
\hline
\end{tabular}

Fuente: Elaboración propia

De acuerdo con estos datos, es un porcentaje muy bajo el de las personas jóvenes con actividades económicas, pues $84 \%$ de los empresarios que tienen a cargo una pyme tienen edades superiores a 31 años.

Siempre en cuanto a los datos de identificación de las características de los microempresarios consultados, $32 \%$ son hombres y $68 \%$ son mujeres.

De las mujeres propietarias de pymes $34 \%$ son casadas, $42 \%$ son solteras, $7 \%$ son viudas y $17 \%$ son separadas.

Del mismo modo, el grado de escolaridad de los microempresarios consultados es muy bajo. Según los datos obtenidos, del total de microempresarios entrevistados, 66 tienen la primaria completa, 12 la secundaria completa y únicamente dos poseen grado universitario.

\section{Venta de bienes o servicios, o ambos, o existencias}

Las actividades comerciales de los microempresarios entrevistados son muy variadas. Como se indica en la siguiente tabla, la cantidad de microempresarios que las realizan y los porcentajes por actividad son los siguientes: 
Cuadro 3

Actividad principal de los microempresarios y microempresarias de Bagaces

\begin{tabular}{|c|c|c|c|}
\hline Actividad principal & $\begin{array}{c}\text { Cantidad } \\
\text { microempresarios }\end{array}$ & Tipo de Actividad & $\begin{array}{c}\% \text { de cada } \\
\text { actividad principal }\end{array}$ \\
\hline Autorr(se sugiere con solo una "r")epuestos & 1 & Comercial & $1 \%$ \\
\hline Abastecedor & 8 & Comercial & $8 \%$ \\
\hline Agricultura orgánica & 2 & Comercial & $2 \%$ \\
\hline Bazar & 4 & Comercial & $4 \%$ \\
\hline Chapia & 3 & Servicio & $3 \%$ \\
\hline Contratista construcción & 2 & Servicio & $2 \%$ \\
\hline Uñas acrílicas & 1 & Servicio & $1 \%$ \\
\hline Elaboración de concentrado & 1 & Industrial & $1 \%$ \\
\hline Funeraria & 1 & Comercial & $1 \%$ \\
\hline Mecánica & 4 & Servicio & $4 \%$ \\
\hline Panadería & 4 & Comercial & $4 \%$ \\
\hline Peluquería & 3 & Servicio & $3 \%$ \\
\hline Productos decoración & 5 & Servicio & $5 \%$ \\
\hline Restaurante & 4 & Comercial & $4 \%$ \\
\hline Sala de belleza & 8 & Servicio & $8 \%$ \\
\hline Sastrería & 2 & Servicio & $2 \%$ \\
\hline Serigrafía & 1 & Servicio & $1 \%$ \\
\hline Souvenir & 3 & Comercial & $3 \%$ \\
\hline Talabartería & 1 & Comercial & $1 \%$ \\
\hline Costura & 7 & Servicio & $7 \%$ \\
\hline Mueblería & 3 & Comercial & $3 \%$ \\
\hline Soldadura & 3 & Servicio & $3 \%$ \\
\hline Tienda & 5 & Comercial & $5 \%$ \\
\hline Venta de artículos plásticos & 1 & Comercial & $1 \%$ \\
\hline Venta de ropa & 7 & Comercial & $7 \%$ \\
\hline Venta de tiliches & 1 & Comercial & $1 \%$ \\
\hline Venta de verduras & 3 & Comercial & $3 \%$ \\
\hline Venta de comida & 8 & Comercial & $8 \%$ \\
\hline Videoclub & 2 & Servicio & $2 \%$ \\
\hline Total & 98 & & $100 \%$ \\
\hline
\end{tabular}

Fuente: Elaboración propia.

Cuadro 4

Tipo de actividad comercial
En resumen, $57 \%$ de las microempresas se dedican a la actividad comercial, $42 \%$ a la actividad de servicios y a actividad industrial, y una microempresa de las 98 , produce y vende sus propios productos.

\begin{tabular}{lc}
\hline Tipo de actividad & Porcentaje \\
\hline Comercial & $57 \%$ \\
Servicios & $42 \%$ \\
Industrial & $1 \%$ \\
\hline Total & $100 \%$ \\
\hline
\end{tabular}

Fuente: Elaboración propia. 
Los datos del cantón de Bagaces contrastan significativamente con los que corresponden al territorio nacional en lo que se refiere a empresas de comercio e industriales. Según el DUIE, las cifras en el caso de empresas comerciales de carácter nacional son de 57\%. Es decir, de $16 \%$ más en comparación con las pymes del cantón de Bagaces. Las empresas industriales del país son $11 \%$ y en el cantón de Bagaces únicamente hay $1 \%$. Y respecto a las empresas comerciales en todo el país hay una representación de $6 \%$, mientras que en el cantón de Bagaces no se encontraron datos sobre esta actividad.

Cuadro 5

Tipo de actividad comercial a nivel nacional

\begin{tabular}{ccccc}
\hline Tamaño/sector & Servicios & Industria & Comercio & TI. \\
\hline Pymes & $42 \%$ & $11 \%$ & $41 \%$ & $6 \%$ \\
\hline
\end{tabular}

Fuente: DUIE 2012, INEC

Del total de los entrevistados 85 dijeron que realizan la venta de sus productos y servicios a crédito y que el periodo de cobro en $70 \%$ está en los rangos de 15 a 30 días. El restante $30 \%$ sobrepasan los 30 días de plazo para cancelar.

\section{Necesidades de capacitación}

En lo que respecta a las necesidades de capacitación propiamente identificadas, estas se dividen en cinco grupos:

Asesoría legal-tributaria. Este término se refiere a la inducción que necesitan recibir los propietarios de pymes en lo que compete a su forma de constitución y a los trámites y procesos que deben llevar a cabo para iniciar sus actividades, tanto en lo legal como en todos los aspectos tributarios que eso conlleva.

Asesoría en modelos de gestión. Los modelos de gestión identificados en las pymes se refieren a la capacidad administrativa y estratégica de la pyme. Entre ellos se distinguen el perfil estratégico, la misión, la visión y los objetivos de la empresa. Además, está la descripción y el entendimiento del proceso administrativo, el proceso de innovación y cómo hacer que los productos tengan un valor agregado, y cuáles estrategias potenciar para llevarlos al mercado.

Asesoría en desarrollo organizacional. Consiste en capacitaciones en servicio al cliente, negociación con proveedores, desarrollo de habilidades de liderazgo, solución creativa de los problemas de la organización, manejo y entendimiento del clima organizacional.

Asesoría tecnológica. Abarca la falta de capacitación en sistemas y herramientas tecnológicas que faciliten las tareas dentro de las pymes.

Condiciones más adecuadas. Cuando se habla de condiciones más adecuadas en realidad esto encierra todas las necesidades descritas, pero también abarca las necesidades de infraestructura y de capital.

Cuadro 6

Necesidades de capacitación de las pymes del cantón de Bagaces

\begin{tabular}{lcc}
\hline Tipo de capacitación & Cantidad de microempresarios & Composición porcentual \\
\hline Asesoría legal-tributaria & 17 & $17 \%$ \\
Asesoría en modelos de gestión & 20 & $20 \%$ \\
Asesoría en desarrollo organizacional & 21 & $21 \%$ \\
Asesoría tecnológica & 19 & $19 \%$ \\
Condiciones más adecuadas & 21 & $21 \%$ \\
\hline TOTALES & 98 & $100 \%$ \\
\hline
\end{tabular}

Fuente: Elaboración propia. 
Del análisis de los datos obtenidos, los aspectos más sobresalientes son los que se refieren a la necesidad de capacitación en modelos de gestión, desarrollo organizacional y condiciones más adecuadas.

En parte, esos datos concuerdan con los de las estadísticas nacionales del MEIC, en que el sector de las pymes representa a(omitir a) cerca de $98 \%$ del parque empresarial del país; $64,6 \%$ son pymes, mayoritariamente de uno hasta tres trabajadores, y 57\% del total de ellas corresponden al área de servicios. En términos generales, las pymes requieren capacitación en materia de acceso a la tecnología, capacidad empresarial y de endeudamiento.

\section{DISCUSIÓN}

\section{Identificación de la empresa}

En la forma de organización predominante de las pymes, como personas físicas, se nota el interés y el empeño empresarial que las caracterizan. Sin embargo, al conocer lo desinformadas que están en materia legal, administrativa y tributaria en cuanto a la puesta en marcha de sus empresas, se evidencia el poco acompañamiento que tienen a la hora de poner en marcha su actividad comercial. Así, muchas de ellas no tienen idea de que deben inscribirse en el Ministerio de Hacienda, de que tienen que tener el permiso de salud o la póliza que las cubra. Con conocimiento de que tienen esas carencias en su experiencia como microempresarios, el propósito de este estudio es hacer conciencia en ellas de que no lo hacen a propósito, sino más bien que esta situación se da por el desconocimiento que tienen y por el poco acompañamiento con que realizan la actividad.

Según los datos obtenidos, más de 50\% de los microempresarios realizan sus actividades comerciales en sus propias casas, lo que les permite disminuir costos en alquiler y tener mayor cercanía con sus familias. No obstante, el realizar los negocios en sus propios hogares ocasiona o permite cierta dispersión al realizar las actividades comerciales. Por ejemplo, pueden incumplir con el principio contable de entidad económica, el cual estipula que la actividad del negocio debe separarse de la de sus dueños o propietarios. Esto demuestra falta de capacitación en gestión de negocios.

No obstante, si se trata de condiciones adecuadas para los microempresarios, el que únicamente $4 \%$ utilicen financiamiento para desarrollar sus actividades, demuestra que no solo hay poca capacitación al respecto, sino también que no hay condiciones apropiadas para que los microempresarios tengan acceso a entidades de financiamiento. Si bien es cierto, el Gobierno ha implementado programas para fortalecer a las pymes, a estas se les sigue tratando como si fueran un sector normal de la economía. Por ejemplo, no se les pueden conceder créditos si no tienen capacidad de pago, y ciertamente nunca lo van a tener. Entonces los parámetros de medición de su necesidad deberían ser, no basados en la capacidad de pago, sino más bien con base en su capacidad productiva.

Asimismo, algunas instituciones colaboran y apoyan a las pymes, pero no tienen los medios necesarios para hacerlo ni fueron creadas para ese propósito, como es el caso del IMAS y el Inamu (INAMU) en lo que respecta a apoyo económico, y del INA en lo que respecta a apoyo técnico y de conocimiento. Las instituciones que realmente fueron creadas para apoyar a las pymes (como el MEIC) no se puede decir que no están haciendo su trabajo, pero se puede afirmar, según los datos obtenidos, que en eso les falta mucho territorio que cubrir.

\section{Características de los microempresarios}

Los propietarios y propietarias de pymes consultados tienen edades entre 31 y 50 años, en su mayoría son mujeres (68\%), y su grado de escolaridad es bastante bajo.

Por ello, de lo anterior se pueden sacar varias conclusiones. La primera es que se trata de familias de escasos recursos que con su espíritu emprendedor se han propuesto sacar avante a sus familias. La segunda es que en su mayoría son mujeres jefas de hogar que necesitan tener una forma de obtener recursos para atender las necesidades básicas de sus familias sin tener que salir 
de sus casas. Además, la difícil situación en la que viven esas mujeres ocasiona que sus opciones de estudio se vean reducidas. Este problema evidencia la necesidad de que haya condiciones más adecuadas para esas personas.

\section{Venta de bienes o servicios, o ambos, o existencias}

La mayoría de las empresas son comerciales o de servicios, lo cual implica que compran los productos para luego comercializarlos. En el caso de las comerciales y en el caso de las de servicios, estas generan algún costo que ellas tienen que asumir con base en los resultados. El problema que se les presenta es que la mayoría de sus ventas las realizan a crédito y no cuentan con formas de financiar sus actividades. En tal caso, tienen que pagar la mercadería con recursos propios y eso les genera pérdida de rentabilidad en su actividad. Claro está que los microempresarios no lo entienden de esa manera, lo cual fortalece la tesis de que hay necesidades de capacitación, puesto que, por ejemplo, se desconocen los modelos de gestión y de desarrollo organizacional del caso.

\section{Necesidades de capacitación}

Parte del objetivo principal de la presente investigación es el de sistematizar las necesidades prioritarias de capacitación de las pymes del cantón de Bagaces, así como establecer algunas características del perfil administrativo de este tipo de empresas.

De acuerdo con la metodología utilizada y por tratarse de una investigación no probabilística, no se pueden generalizar los resultados que se esperan; no obstante, estos dan un panorama de cuáles son realmente las necesidades si se comparan dichos resultados con los datos de estadísticas de desarrollo de dicho cantón.

Dada la importancia de que se administren adecuadamente las pymes es necesario aplicar en ellas aquellos conceptos que contribuyan a mejorar la administración.
Para Soto Pineda (2003: 48), "las empresas en el futuro serán organizaciones pequeñas con empresarios jóvenes para favorecer su adaptabilidad y sustentabilidad, y los productos y servicios tendrán valor de acuerdo con la inteligencia aplicada que tienen incorporada.

Independientemente del tipo de empresa de que se trate, el acto de administrar conlleva planear, organizar, dirigir y controlar en las etapas indispensables para alcanzar el éxito empresarial. Sin embargo, el caso de las pymes no es la excepción, pues dueños o gestores necesitan estar capacitados en procesos administrativos para poder hacerles frente de buena forma a las exigencias de su negocio.

Con base en la definición conceptual de Soto Pineda, en la que se enfatiza que las pymes en el futuro van a estar formadas por jóvenes para favorecer la adaptabilidad y la sustentabilidad, se diría que esto no se ha encontrado en el cantón de Bagaces, pues es notorio que ahí los jóvenes no desarrollan dichos procesos. Más bien, como se ha visto, la iniciativa de crear y administrar pymes la tienen personas adultas, mayores de 31 años. Pero, ¿qué se está haciendo para crear una cultura empresarial en la juventud? Aquí se presenta la primera debilidad en cuanto a estrategias de pymes. Por ningún lado se encuentra ni promoción ni afinamiento, ni enamoramiento o interés de los jóvenes en el tema. Por ende, la pregunta es: ¿cuál es la estrategia que debieran seguir las entidades comprometidas con las pymes?

Por su parte, es difícil a veces pensar que las instituciones van a ir a la puerta de los microempresarios, pues resulta un poco complicado ubicarlos y tener que ir a buscarlos, pero hace falta que todos los programas del Gobierno que buscan fortalecer a las pymes sean promocionados por medios de difusión muy activos. Claro está que se ha hecho un gran esfuerzo por publicitar y dar a conocer esos programas, aunque cuando se revisa a estas microempresas se cae en la cuenta de que en su mayoría no tienen acceso ni a la tecnología ni, en muchos casos, a medios de difusión.

Hablar de necesidad de una capacitación integral es ya tratar de estructurar un plan de capacitación en el que se contemple desde los 
principios básicos de la administración hasta qué se debe hacer y cómo administrar los recursos que se van generando.

Desconcierta cuando al identificar a una microempresa y conocer sus ideales y sus capacidades se nota que sus representantes no cuentan con la suficiente información o conocimiento de cómo administrarla, cómo controlarla o cuáles son los procesos de registro y sus responsabilidades.

Lo mejor que se puede hacer por los microempresarios es darles la oportunidad de aprender y para eso proponerles talleres y cursos, y animarlos a que utilicen los programas. La administración requiere capacitación y necesita ser autodirigida, entendiendo al mismo tiempo que la capacidad de aprendizaje depende del nivel profesional con que se le oriente.

Así, la incorporación de nuevas tecnologías es imprescindible para que las pymes mejoren su productividad y, en consecuencia, aumenten las ventajas competitivas. En ese tenor, se considera que las pymes desempeñarán un papel importante, ya que influyen directamente en el mercado laboral y dinamizan la creación de empleos; pero, por otro lado, pueden contribuir a la pérdida de este, si su situación no es favorable.

La capacitación es la base fundamental de un programa amplio de administración de la calidad. Cuando se habla de calidad esta tiene que ver con máquinas, materiales y medidas; pero también puede orientarse al desempeño empresarial, es decir, que se pueden diseñar programas de capacitación para promover un trabajo de mayor calidad por parte de las empresas.

La capacitación es uno de los instrumentos más importantes con los que se cuenta para la transformación de una empresa, y tiene como propósito fundamental mejorar el rendimiento actual y futuro de la fuerza de trabajo. Esto se logra mediante la superación de los conocimientos básicos, el perfeccionamiento de las habilidades específicas y la inducción y adecuación de las actitudes de las personas.

No obstante, la capacitación y el desarrollo no se pueden aplicar con éxito si solo se cuenta con buenos deseos y nobles intenciones.
Es necesario diseñar y poner en práctica programas específicos, los cuales deben apoyarse en una clara determinación de necesidades, como han sido planteadas de alguna manera en el presente escrito.

Para finalizar el presente artículo, quedan claramente identificados los problemas que tienen las pymes del cantón de Bagaces, y también queda claro cuáles son las necesidades prioritarias de capacitación identificadas. Sin embargo, es irresponsable decir cuál es la estructura que debería tener el programa de capacitación por desarrollar, porque eso requiere un estudio más minucioso en lo que se refiere a esta materia. Lo que sí se puede decir es que cualquier programa aplicable al caso debe llevar las variables identificadas y ser un plan de capacitación integral.

\section{Referencias bibliográficas}

Best, J. (1982). Cómo investigar en educación. Madrid, España: Prentice Hall Inc.

Córdoba, F. G. (2004). Recomendaciones metodológicas para el diseño de un cuestionario. México: LIMUSA.

Elecciones, T. S. (2006). Bagaces, Guanacaste.

Grande Esteban, I. \& Abascal Fernández, E. (2011). Fundamentos y técnicas de investigación comercial. España: ESIC EDITORIAL.

Instituto de Formación y Estudios de Democracia. (2006). Bagaces.

MEIC. (6 de 10 de 1997). Definición oficial de Pyme. La Gaceta Decreto Ejecutivo Número 26.426, pág. 11.

MEIC. (2013). Estado de Situación de las Pymes en Costa Rica, 2013. San José, Costa Rica.: Ministerio de Economía Industria y Comercio.

Municipalidad de Bagaces. (29 de 06 de 2013). Breve Reseña Histórica del Cantón de Bagaces. Obtenido de Breve Reseña Histórica del Cantón de Bagaces: www.bagaces. go.cr

Namakforoosh, M. N. (2005). Metodología de la investigación. México DF: Editorial LIMUSA, S.A de C.V. 
Nogales, Á. F. (2004). Investigación y técnicas de mercado. Madrid, España: ESIC EDITORIAL.

Soto Pineda, E. (2003). Las PYMES ante el desafío del siglo XXI: los nuevos mercados globales. México: Thompson Editores, S.A.

Torres, C. A. (2006). Metodología de la investigación: para administración, economía, humanidades. México: Pearson Educación de México, S.A de C.V. 
